\title{
LOW FREQUENCY IMAGING AND THE NON-ISOPLANATIC ATMOSPHERE
}

\author{
C. R. SUBRAHMANYA \\ Tata Institute of Fundamental Research, Poona University Campus, Post \\ Bag 3, Pune 411 007, India
}

\begin{abstract}
At low frequencies, the typical fields of view over which imaging is required is several degrees across, for which (a) the visibility and brightness distribution cannot be assumed to be related by a two-dimensional Fourier Transform, and (b) the propagation delays introduced by the atmosphere cannot be assumed constant within the field of view. These are adequately represented in a method discussed in this paper. Although compute-intensive, the method is suitable for implementation on parallel processors.
\end{abstract}

\section{INTRODUCTION}

The significant increase in dynamic ranges achieved in radio maps over the last decade is due to the success of self-calibration techniques. (Schwab 1980; Cornwell and Wilkinson 1981; Noordam and de Bruyn 1982). These methods assume that the primary beam of each antenna at ionospheric heights (or the array extent) corresponds to a region of ionosphere within which propagation delays can be assumed constant. The ionosphere is said to be isoplanatic in such a region. The assumption of isoplanaticity is questionable at low frequencies where the field of view of practical antennas can be a few tens of kilometres at typical ionospheric heights. In the case of arrays like the VLA or GMRT, the primary beams of individual antennas at metre-wavelengths overlap strongly at ionospheric heights. A generalization of self-calibration for such cases has been described in Subrahmanya(1990). An algorithm for image reconstruction using this scheme is presented below.

\section{SELF-CALIBRATION}

In this Section, we first explain the notation followed in this paper and state the self-calibration algorithm in our notation. A model brightness distribution $B(x, y)$ is described in terms of a number of point sources in the field of view, e.g., obtained by using CLEAN for deconvolution. Thus, 


$$
B(x, y)=\sum_{k} A_{k} \delta\left(x-x_{k}, y-y_{k}\right)
$$

where $A_{k}$ is the amplitude of the "point-component" at $\left(x_{k}, y_{k}\right)$. We now define $\xi_{k}$ as the contribution to visibility from this point component(e.g., see Thomson et al. 1986),

$$
\xi_{k}=A_{k} e^{-2 \pi i\left(u x_{k}+v y_{k}+w \sqrt{1-x_{k}^{2}-y_{k}^{2}}\right)}
$$

It may be noted that the effect of the "w-term" has been incorporated into the above expression. Similarly, any known calibration terms can also be absorbed into the definition of $\xi_{k}$. However, this is not true of the atmospheric effects and time-varying instrumental effects which cannot be calibrated directly. In such cases, there will be an unknown complex gain term which needs to be determined at intervals of seconds to minutes. For simplicity, we will ignore at this stage fluctuations in amplitude gain and concentrate on the phase distortions which are more important for the atmospheric effects under discussion. (As we shall see later, this assumption is not a limiting factor for our algorithm.) Under the assumption of isoplanaticity, all the atmospheric effects (as well as the instrumental effects) can be described adequately by a single phase term for each antenna. Assuming that a phase $\phi_{p}$ is introduced by such effects in the signal arriving at antenna $p$, we may write the model visibility (isoplanatic case) as

$$
V_{p q}^{i, \rho}=e^{i\left(\phi_{r}-\phi_{q}\right)} \sum_{k} \xi_{k}
$$

where $V_{p q}$ is a visibility corresponding to the baseline determined by antennas $p$ and $q$. The self-calibration consists in the determination of the phases $\phi$ by minimising the mean square difference between the observed and model visibilities.

It is implicitly assumed above that either the antenna beam intercepted by the ionosphere or the baseline is smaller than a typical isoplanatic patch. For arrays like the VLA or GMRT, neither of these situations prevail and it is essential to model the visibility by considering different phases for different directions within the primary beam of each antenna. In such cases, the model visibility can be written as

$$
V_{p q}^{n o n}=e^{i\left(\phi_{p}-\phi_{q}\right)} \sum_{k} \xi_{k} e^{i\left[\psi_{k}(p)-\psi_{k}(q)\right]}
$$

Note the appearance of phases inside the summation in the above equation, which implies that the effective gain is no longer a pure phase although we ignored amplitude gain fluctuations. The assumption of constant 
amplitude gasn could have been relaxed without adding extra computing load since amplitude and phase calibrations are not separable in the non-isoplanatic case.

In the most general case, one divides the primary beam of each antenna into a certain number of cells within which the ionosphere can be assumed isoplanatic, and solves for the phases associated with all the antennas in the array (Schwab 1984). However, when the antenna beams overlap appreciably at the ionospheric heights as in the case of GMRT or VLA, this method introduces an undesirably large number of artificial parameters to be solved in a system of nonlinear equations. This can be avoided by partitioning the region of ionosphere spanned by the entire array into cells to which a phase is attributed (Subrahmanya 1990). Thus, we may write

$$
\psi_{k}(p)=\sum_{l} \omega_{l}\left(p ; x_{k}, y_{k}\right) \theta_{l}
$$

where $\theta_{l}$ are the phases associated with the ionospheric cells and the $\omega_{l}$ are the weights defining an interpolation scheme for specifying the $\psi$ in terms of the $\theta$. It may be noted that such a formalism assumes a standard height at which the ionospheric cells are located, implying a thin-screen approximation to the ionosphere. More complicated situations may require the description of the ionosphere in terms of multiple thin-screens. The exact expressions for $\omega$ depend on these details and the geometry. If necessary, the height of the ionospheric screen can be easily introduced an additional parameter to be determined along with the phases. However, since the effective ionospheric height varies very slowly in comparison with the phases, the additional computational load introduced by the need to solve for the heights is negligible.

\section{IMAGE RECONSTRUCTION WITH PHASE CORRECTION}

We now describe briefly an algorithm for image reconstruction incorporating the above features. This is similar to the AIPS task ' $M X$ ' in many respects.

(a) After applying known gain corrections, grid the data and use CLEAN or similar deconvolution method to arrive at a model $B(x, y)$. The model need only be good to a moderate dynamic range of 10 or so.

(b) From the model brightness distribution, find $\xi_{k}$ and formulate the model visibility $V_{p q}^{n o n}$ in terms of $\Delta \phi, \Delta \theta$.

(c) Find the least-squares solution for $\Delta \phi, \Delta \theta$ by minimising the sum of squares of residual visibilities. This leads to a set of nonlinear simultaneous equations which can be solved iteratively by Newton-Raphson method (Subrahmanya 1990). Note that the instrumental effects $\phi$ are explicitly obtained without mixing with the $\theta$ which describe ionospheric effects. This is of particular significance since the values obtained from a calibration session are a good approximation to the instrumental phases, but not the ionospheric phases. 
(d) The model visibility is now re-calculated using the updated phases and the residual visibilities derived from them. The steps (a)-(d) constitute a major cycle of iteration in which visibilities are updated accurately. In particular, the w-term has automatically been accounted for.

(e) The residual visibilities are gridded, substituted in the first step to find corrections to model brightness distribution and the above major iteration cycle is repeated until the residual visibilities appear noise-like.

\section{DISCUSSIQN}

In the method suggested for self-calibration, the instrumental effects are still antenna-based as in the normal self-calibration which assumes isoplanaticity. On the other hand, the ionospheric effects have been parametrised into a separate set which are explicitly related to the ionosphere. Thus it is possible to use different effective integration times for instrumental and atmospheric effects. This is useful since the two effects generally have very different time scales of variability.

In the isoplanatic case, one can determine a set of phases with which the observed visibilities can be corrected (re-calibrated) such that they can lead to the correct image after proper deconvolution. In the present case, such corrections depend explicitly on the image itself since they are a combination of different corrections in various directions within the antenna beams. With such a baseline-dependence, it is not useful to describe the results as gain correction to observed visibilities. The only meaningful interpretation is that the suggested algorithm leads to a model $B(x, y)$ and a set of phases consistent with the observations. It is thus important to note that each major cycle(except the first) should only begin with the residual visibilities but not corrected visibilities which would have been equally acceptable in a normal selfcalibration restricted to antenna-based solutions.

By relating the parameters explicitly to the ionosphere, it becomes more convenient to use any known properties of ionospheric effects to improve the image reconstruction. For instance, the solutions to different time-slices can be used to formulate a model in which the phases result from moving ionospheric patches whose velocities could be solved for. Using realistic models like this, one can effectively increase the integration time available for determining the ionospheric phases and thus improve the accuracy. However, it is important to note that the parameters have so far been related to the ionosphere only geometrically, not physically. Further work is required in order to use the interferometric observations for increasing our understanding of the ionosphere, or to translate the known statistical properties of the ionosphere into interferometric errors so that more appropriate models could be used.

Although the algorithm suggested in the previous section is heavily compute-intensive, most of the computation consists of calculating the model visibilities and the solution of nonlinear equations. Since we require only a moderate dynamic range for CLEAN, the relative computing load for this step is small. The algorithm is well suited for implementation on special purpose machines employing parallel processing technique (e.g. Kulkarni and Subrahmanya, this Conference.) In such a system, different time-slices of data could be assigned to different processors which would be independently 
updating visibilities and estimating phases for their respective timeslices of data. Since the computations for different time-slices are independent, there is no need for fast exchange of intermediate results amont the processors during computations. This makes the algorithm very efficient for parallel processing systems.

\section{REFERENCES}

Cornwell, T. J. and Wilkinson, P. N. 1981, M.N.R.A.S., 196, 1067.

Noordam, J. E. and deBruyn, A. G. 1982, Nature, 299, 597.

Schwab, F. R. 1980, Proc. Soc.of Photo-Optical Instrumentation Engineers, $231,18$.

Schwab, F. R. 1984,Astron. J. 89, 1076.

Subrahmanya, C. R. 1990, in URSI/IA U Symposium on Radio Astronomical Seeing, eds. Wang Shouguan and Yang Yi-Pei, Chinese Acad. Sci., Beijing.

Thomson, A. R., Moran, J. M. and Swensen, G. W. 1986, Interferometry and Synthesis in Radio Astronomy, Wiley, Section 4.1.

Ray Norris: Have you considered the use of Kalman filtering to separate the ionospheric effects from other effects, given the known statistical differences? Subrahmanya: No. Ionospheric effects have been formally separated from other effects which are treated as being antenna specific. One can further examine the statistics by considering sets of ionospheric phases obtained over a period of time. But I don't know how to translate such effects into constraints on the least- squares estimates of phases.

John Baldwin: The thickness of the F-region exceeds its mean height so I think your ionosphere model will need to be more complicated than one thin screen. Is that a problem for your method?

Subrahmanya: Multiple thin screens can be accommodated easily. However, I am not sure if it is practical to have more than 2-3 screens. I prefer to be conservative in increasing the number of parameters without further input from physical considerations.

John Findlay: There are regular traveling disturbances in both the $\mathrm{E}$ and $\mathrm{F}$ regions of the lower flux. These may cause phase errors in the GMRT at lower frequencies and will not be described by the random disturbances discussed. But there is much knowledge of these in India - so you should be OK! 